\title{
Field Efficacy of Newer Insecticides and Neem Products against Scirtothrips dorsalis on Grapes, Vitis vinifera L. (cv. Thompson Seedless)
}

\author{
Nagaraj R. Patil ${ }^{1 *}$, A.M. Nadaf ${ }^{1}$, B.S. Sagar ${ }^{2}$ and B.R. Sahithya ${ }^{2}$ \\ ${ }^{1}$ Department of Horticultural Entomology, College of Horticulture, University of Horticultural \\ Sciences, Bagalkot - 587104, Karnataka, India \\ ${ }^{2}$ Department of Fruit Science, College of Horticulture, University of Horticultural Sciences, \\ Bagalkot - 587104, Karnataka, India \\ *Corresponding author
}

\begin{tabular}{|c|c|}
\hline \multicolumn{2}{|r|}{ A B S T R A C T } \\
\hline & \multirow{6}{*}{ 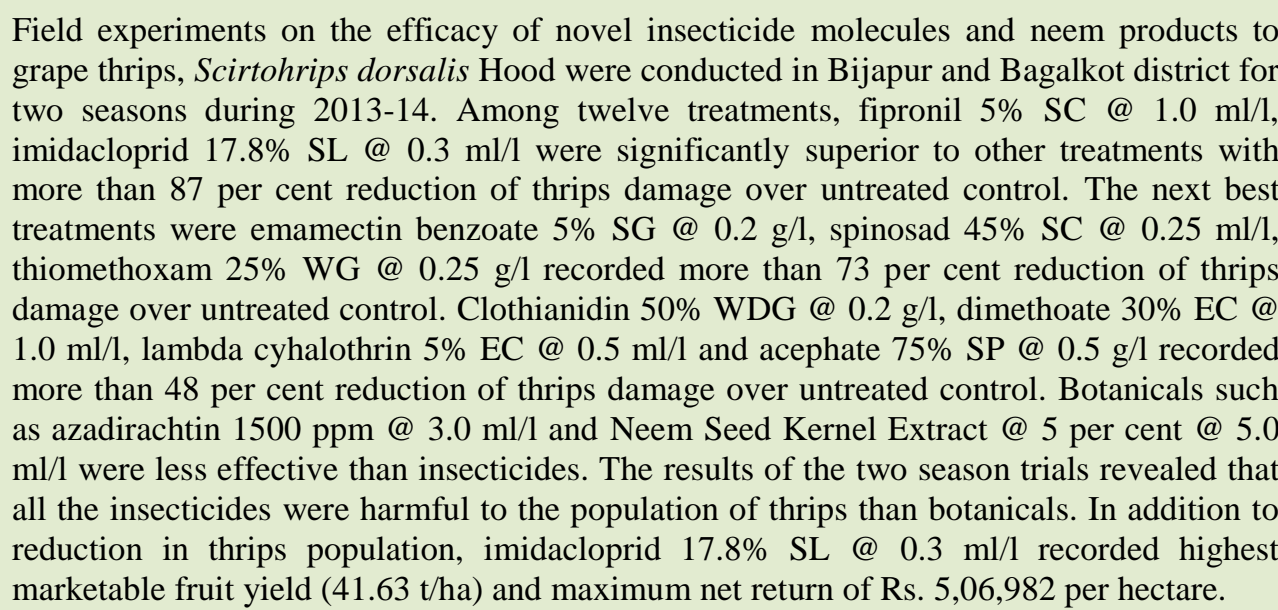 } \\
\hline & \\
\hline $\begin{array}{l}\text { Grapes, Scirtothrips } \\
\text { dorsalis, } \\
\text { Imidacloprid. }\end{array}$ & \\
\hline Article Info & \\
\hline $\begin{array}{l}\text { Accepted: } \\
\text { 10 July } 2017 \\
\text { Available Online: } \\
\text { 10 September } 2017\end{array}$ & \\
\hline & \\
\hline
\end{tabular}

\section{Introduction}

Grape (Vitis vinifera L.) is one of the most important fruit crops of temperate zone, which has acclimatized to sub-tropical and tropical agro climatic conditions prevailing in the Indian sub-continent (Bose et al., 1999). In India, grape is commercially grown in an area of about $1,16,000$ hectares with annual production of $24,83,000$ tonnes with a productivity of 21.1 metric tonnes per hectare (Anon., 2013). In India, grapes growing states are Maharashtra, Karnataka, Andhra Pradesh and Tamil Nadu.
Maharashtra stands first with 29,800 hectares followed by Karnataka having 8,200 hectares with an annual production of 7.79 and 2.28 lakh tonnes, respectively.

Bijapur is one of the major districts of Karnataka in grape cultivation (6137 ha) with an annual production of 1.22 lakh tonnes.

Of the total production, nearly 60 per cent goes for the production of raisins and 40 per cent is used for table purpose (Anon., 2012). 
The fresh grape berries are good source of sugars, carbohydrates, vitamins, proteins and minerals. Fruits are used for table purpose, wine, juice, raisins and canning. Fresh and dried fruits have various uses in Ayurvedic and Unani medicine.

The fruits are considered to be laxative, stomachic, diuretic and cooling agents. The juice of unripe berries is used as astringent in throat infections.

Insect pests are the important production constraints in grape cultivation next to diseases. Bournier (1977) listed 132 insect pests attacking grapes in the world. In grape, 85 species of insect pests have been reported in India (Atwal and Dhaliwal, 2005), but only a few of them are of potential threat and have gained the major importance as far as loss in yields caused by them are concerned. Among them, grape thrips (Scirtothrips dorsalis Hood) is of much importance and the most damaging pest species, infestation in grape (leaves) take whitish hue, acquire a withered appearance and then turn brown. Leaves ultimately curl up and drop off. Keeping in view the economic importance of this insect pest, the present study was undertaken to control through chemical and botanical means.

Generally, conventional insecticides belonging to organophosphates and synthetic pyrethroides are used to control the thrips in grapes (Sunitha and Jagginavar, 2008).

\section{Materials and Methods}

Field experiment was conducted at progressive former field, Khedagi, Bijapur district after April and October pruning (2013 and 2014) in randomized block design with twelve treatments and three replications to determine the efficacy of new molecules of insecticide at various doses against thrips infesting grape Vitis vinifera L. (cv. Thompson Seedless). The treatments were azadirachtin 1500ppm @ $3.0 \mathrm{ml} / \mathrm{l}$, neem seed kernel extract (NSKE) 5\% @ 5.0 ml/l, Fipronil 5\%SC @ $1.0 \mathrm{ml} / 1$, emamectin benzoate 5\%SG @ $0.2 \mathrm{~g} / \mathrm{l}$, spinosad 45\% SC @ $0.25 \mathrm{ml} / \mathrm{l}$, imidacloprid 17.8\%SL @ 0.3 $\mathrm{ml} / \mathrm{l}$, thiomethoxam 25\%WG @ $0.25 \mathrm{~g} / \mathrm{l}$, clothianidin 50\%WDG @ $0.20 \quad \mathrm{~g} / \mathrm{l}$, dimethoate 30\%EC @ $1.0 \mathrm{ml} / \mathrm{l}$, lambda cyhalothrin 5\%EC @0.5 ml/l and acephate $75 \% \mathrm{SP} @ 0.5 \mathrm{~g} / \mathrm{l}$. The respective chemical treatments were sprayed on grape as and when thrips population reached peak in vegetative stage two (fifteen days after pruning) using knapsack sprayer with high volume fitted hollow cone nozzle. Altogether, two applications were made at 10 days intervals during both April and October pruning after. Observations on the number of thrips were recorded one day prior to the spray and three and ten days after the spray.

Pre-treatment and post treatment counts on 10 randomly selected shoots were taken by gently beating the shoots, five times against a black cardboard sheet and the number of thrips present on the black cardboard sheet were counted. Number of berries damaged by the total number of berries was recorded from 10 bunches per vine were randomly selected from each vine at after ten days of second spray during October pruning and then berry damage (\%) was calculated. The yield was recorded on the net plot area basis which was converted to $\mathrm{kg} / \mathrm{ha}$ for statistical interpretations. The monetary returns and incremental cost-benefit ratios of treatments were assessed based on the yield and cost of plant protection. The data obtained from field experiments were analysed using the analysis of variance for randomized block design (Gomez and Gomez, 1984). Following ANOVA, differences between datasets were determined using least significant difference at $\mathrm{P}=0.05$ in all instances. 


\section{Results and Discussion}

\section{Thrips on shoots after April and October pruning}

Table 1 depicts the grape thrips, Scirtothrips dorsalis population was uniform in all the treatments before spray as treatment difference was non-significant ranging from 9.00 to 10.60 thrips per shoot during after April pruning 2013 (Table 1). There was a significant reduction in thrips population after spraying of the insecticides over untreated control. Fipronil $\left(\mathrm{T}_{3}\right)$ and Imidacloprid $\left(\mathrm{T}_{6}\right)$ recorded significantly lower infestation of thrips (1.56 and 1.66 thrips/shoot respectively). The results are in accordance with Jyoti et al., (2013) who reported that application of fipronil $\left(\mathrm{T}_{3}\right)$ and imidacloprid $\left(\mathrm{T}_{6}\right)$ were found to be effective in reducing thrips number in mulberry. The next best treatment Emamectin benzoate $\left(\mathrm{T}_{4}\right) \quad(3.00$ thrips/shoot), Spinosad ( $\mathrm{T}_{5}$ ) (3.46 hrips/shoot) and Thiomethoxam $\left(\mathrm{T}_{7}\right)$ (3.70 thrips/shoot) and were on par with each other followed by Clothianidin $\left(\mathrm{T}_{8}\right)$ (4.83 thrips/shoot). These results are in conformity with the reports of Kanara and Acharya (2014), who reported the effective of emamectin benzoate and spinosad in reducing thrips population in rose. The other treatments, Dimethoate $\left(\mathrm{T}_{9}\right) \quad(5.56$ thrips/shoot), Lambda Cyhalothrin $\left(\mathrm{T}_{10}\right)$ (5.90 thrips/shoot) and Acephate $\left(\mathrm{T}_{11}\right) \quad(6.80$ thrips/shoot) which were statistically on par with each other. The botanical produces Azadirachtin $\left(\mathrm{T}_{1}\right) \quad(8.20$ thrips/shoot $)$ and Neem seed kernel extract (NSKE) 5\% (T2) (8.43 thrips/shoot) were found to be statistically superior over untreated control (11.43 thrips/shoot). These results are close to the findings of Bhargava and Bhatnagar (2005) and Suresh et al., (2006) who reported that, NSKE and nimbicidine were found effective in controlling the thrips population but at lower rate as compared to chemical insecticides.
A similar trend was noticed in the treatments at 10 DAS of first spray and 3,10 DAS of second spray. Fipronil $\left(\mathrm{T}_{3}\right)$ and imidacloprid $\left(\mathrm{T}_{6}\right)$ were recorded significantly lower infestation in all the observations and was found superior to the untreated control, Emamectin benzoate $\left(\mathrm{T}_{4}\right)$, Spinosad $\left(\mathrm{T}_{5}\right)$ and Thiomethoxam $\left(\mathrm{T}_{7}\right)$ in reducing the thrips damage.

The results of the second field experiment conducted during after October pruning 201314 (Table 2) also provided a comparable trend of reduction in the thrips damage with respect to different treatments. Fipronil $\left(\mathrm{T}_{3}\right)$ and imidacloprid $\left(\mathrm{T}_{6}\right)$ also provided a significant decrease in the level of thrips infestation in both after April and October pruning trials, compared to the untreated control.

\section{Effect on berry damage}

The plots with newer insecticides registered significantly low incidence of neem product in comparison to farmers practices (Table 3). Minimum fruit damage of $21.89 \%$ and 24.60 $\%$ (on number basis) was recorded in the treatment fipronil $\left(\mathrm{T}_{3}\right)$ and imidacloprid $\left(\mathrm{T}_{6}\right)$.

The results are in line with findings of Sunitha et al., (2008) who reported significantly less bunch infestation in neonicotinoid treated plots. These three treatments i.e. emamectin benzoate $\left(\mathrm{T}_{4}\right)$, Spinosad $\left(\mathrm{T}_{5}\right)$ and Thiomethoxam $\left(\mathrm{T}_{7}\right)$ were at par with each other in minimizing the thrips incidence $(30.81,31.34$ and 33.70 percent berry damage respectively) followed by Clothianidin $\left(\mathrm{T}_{8}\right)$ with $38.34 \%$ fruit damage. The treatments Dimethoate $\left(\mathrm{T}_{9}\right)$, Lambda Cyhalothrin $\left(\mathrm{T}_{10}\right)$ and Acephate $\left(\mathrm{T}_{11}\right)$ were also found to be moderately effective with 48.94, 49.49 and 47.92 percent fruit damage respectively. Among the botanical products evaluated, Azadirachtin $\left(\mathrm{T}_{1}\right)(56.43 \%$ berry damage) and Neem seed kernel extract 
(NSKE) (T2) (60.15\% berry damage) proved relatively inferior in managing the thrips infestation. Highest thrips damage on berries was noticed on untreated control $\left(\mathrm{T}_{12}\right)$.

Table.1 Efficacy of new molecules of insecticides on grape thrips cv. Thompson Seedless

(Vitis vinifera L.) after April pruning, 2013-14 (on shoots)

\begin{tabular}{|c|c|c|c|c|c|c|c|c|}
\hline \multirow{3}{*}{$\begin{array}{l}\text { Tr. } \\
\text { No. }\end{array}$} & \multirow{3}{*}{ Treatments } & \multicolumn{5}{|c|}{ No. of $S$. dorsalis per shoot* } & \multirow[b]{3}{*}{ Mean } & \multirow{3}{*}{$\begin{array}{l}\text { Per cent } \\
\text { reduction } \\
\text { over UTC }\end{array}$} \\
\hline & & \multirow[b]{2}{*}{ PTC } & \multicolumn{2}{|c|}{ First Spray } & \multicolumn{2}{|c|}{ Second Spray } & & \\
\hline & & & 3 DAS & 10 DAS & 3 DAS & 10 DAS & & \\
\hline $\mathrm{T}_{1}$ & Azadirachtin 1500ppm @ 3.0 ml/1 & 9.82 & $\begin{array}{c}8.20 \\
(2.95) \mathrm{e}\end{array}$ & $\begin{array}{c}9.00 \\
(3.08) \mathrm{e}\end{array}$ & $\begin{array}{c}8.50 \\
(3.00) \mathrm{e}\end{array}$ & $\begin{array}{c}7.60 \\
(2.85) \mathrm{e}\end{array}$ & 8.32 & 29.49 \\
\hline $\mathrm{T}_{2}$ & NSKE 5\% @ 5.0 ml/1 & 9.62 & $\begin{array}{c}8.43 \\
(2.99) \mathrm{e}\end{array}$ & $\begin{array}{c}9.16 \\
(3.11) \mathrm{e}\end{array}$ & $\begin{array}{c}8.76 \\
(3.04) \mathrm{e}\end{array}$ & $\begin{array}{c}8.16 \\
(2.94) \mathrm{e}\end{array}$ & 8.62 & 26.94 \\
\hline $\mathrm{T}_{3}$ & Fipronil 5\%SC @ 1.0 ml/l & 10.03 & $\begin{array}{c}1.56 \\
(1.42) \mathrm{a}\end{array}$ & $\begin{array}{c}1.90 \\
(1.55) \mathrm{a}\end{array}$ & $\begin{array}{c}1.20 \\
(1.30) \mathrm{a}\end{array}$ & $\begin{array}{c}0.70 \\
(1.09) \mathrm{a}\end{array}$ & 1.43 & 87.88 \\
\hline $\mathrm{T}_{4}$ & Emamectin benzoate 5\%SG @ $0.2 \mathrm{~g} / 1$ & 9.00 & $\begin{array}{c}3.00 \\
(1.87) \mathrm{b}\end{array}$ & $\begin{array}{c}3.30 \\
(1.95) \mathrm{b}\end{array}$ & $\begin{array}{c}2.83 \\
(1.81) \mathrm{b}\end{array}$ & $\begin{array}{c}2.53 \\
(1.73) \mathrm{b}\end{array}$ & 2.91 & 77.33 \\
\hline $\mathrm{T}_{5}$ & Spinosad 45\%SC @ 0.25 ml/l & 9.50 & $\begin{array}{c}3.46 \\
(1.99) b\end{array}$ & $\begin{array}{c}3.40 \\
(1.97) \mathrm{b}\end{array}$ & $\begin{array}{c}3.00 \\
(1.87) \mathrm{b}\end{array}$ & $\begin{array}{c}2.66 \\
(1.78) b\end{array}$ & 3.13 & 73.47 \\
\hline $\mathrm{T}_{6}$ & Imidacloprid 17.8\%SL @ 0.3 ml/1 & 9.60 & $\begin{array}{c}1.66 \\
(1.46) \mathrm{a}\end{array}$ & $\begin{array}{c}1.90 \\
(1.55) \mathrm{a}\end{array}$ & $\begin{array}{c}1.50 \\
(1.41) \mathrm{a}\end{array}$ & $\begin{array}{c}1.20 \\
(1.30) \mathrm{a}\end{array}$ & 1.56 & 86.77 \\
\hline $\mathrm{T}_{7}$ & Thiomethoxam 25\%WG @ $0.25 \mathrm{~g} / 1$ & 10.00 & $\begin{array}{c}3.70 \\
(2.05) \mathrm{b}\end{array}$ & $\begin{array}{c}4.13 \\
(2.15) \mathrm{b}\end{array}$ & $\begin{array}{c}3.53 \\
(2.00) \mathrm{b}\end{array}$ & $\begin{array}{c}3.13 \\
(1.90) \mathrm{b}\end{array}$ & 3.62 & 69.32 \\
\hline $\mathrm{T}_{8}$ & Clothianidin 50\%WDG @ $0.2 \mathrm{~g} / 1$ & 10.30 & $\begin{array}{c}4.83 \\
(2.30) \mathrm{c}\end{array}$ & $\begin{array}{c}5.81 \\
(2.51) \mathrm{c}\end{array}$ & $\begin{array}{c}5.11 \\
(2.37) \mathrm{c}\end{array}$ & $\begin{array}{c}4.91 \\
(2.32) \mathrm{c}\end{array}$ & 5.16 & 56.27 \\
\hline $\mathrm{T}_{9}$ & Dimethoate 30\%EC @ $1 \mathrm{ml} / \mathrm{l}$ & 9.52 & $\begin{array}{c}5.56 \\
(2.46) \mathrm{d}\end{array}$ & $\begin{array}{c}7.13 \\
(2.76) d\end{array}$ & $\begin{array}{c}6.53 \\
(2.65) \mathrm{d}\end{array}$ & $\begin{array}{c}5.93 \\
(2.59) \mathrm{d}\end{array}$ & 6.55 & 44.49 \\
\hline $\mathrm{T}_{10}$ & Lambda cyhalothrin 5\%EC @ $0.5 \mathrm{ml} / 1$ & 9.60 & $\begin{array}{c}5.90 \\
(2.53) \mathrm{d}\end{array}$ & $\begin{array}{c}7.10 \\
(2.76) \mathrm{d}\end{array}$ & $\begin{array}{c}6.60 \\
(2.66) \mathrm{d}\end{array}$ & $\begin{array}{c}6.90 \\
(2.66) \mathrm{d}\end{array}$ & 6.62 & 43.89 \\
\hline $\mathrm{T}_{11}$ & Acephate 75\% SP @ 0.5 g/l & 10.60 & $\begin{array}{c}6.80 \\
(2.70) \mathrm{d}\end{array}$ & $\begin{array}{c}7.20 \\
(2.77) \mathrm{d}\end{array}$ & $\begin{array}{c}6.40 \\
(2.63) \mathrm{d}\end{array}$ & $\begin{array}{c}6.80 \\
(2.60) \mathrm{d}\end{array}$ & 6.75 & 42.79 \\
\hline $\mathrm{T}_{12}$ & Untreated control & 10.30 & $\begin{array}{c}11.43 \\
(3.46) \mathrm{f}\end{array}$ & $\begin{array}{c}12.00 \\
(3.54) \mathrm{f}\end{array}$ & $\begin{array}{c}12.50 \\
(3.61) \mathrm{f}\end{array}$ & $\begin{array}{c}11.30 \\
(3.44) f\end{array}$ & 11.80 & - \\
\hline & $\mathrm{SE} \mathrm{m} \pm$ & NS & 0.08 & 0.08 & 0.09 & 0.09 & & \\
\hline & $\mathrm{CD}$ at $5 \%$ & NS & 0.24 & 0.24 & 0.25 & 0.26 & & \\
\hline & $\mathrm{CV}(\%)$ & NS & 5.93 & 5.70 & 6.30 & 6.90 & & \\
\hline
\end{tabular}

Figures in the parentheses are $\sqrt{\mathrm{x}+0.5}$ transformed values

Means followed by same letter do not differ significantly by DMRT ( $\mathrm{P}=0.05)$, *Mean of thirty shoots

PTC - Pretreatment count per shoot

DAS - Day After Spraying

UTC - Untreated control 
Table.2 Efficacy of new molecules of insecticides on grape thrips cv. Thompson Seedless (Vitis vinifera L.) after Ocotober pruning, and 2013-14 (on shoots)

\begin{tabular}{|c|c|c|c|c|c|c|c|c|c|}
\hline \multirow[b]{3}{*}{$\begin{array}{l}\text { Tr. } \\
\text { No. }\end{array}$} & \multirow[b]{3}{*}{ Treatments } & \multicolumn{5}{|c|}{ No. of $S$. dorsalis per shoot on different days* } & \multirow[b]{3}{*}{ Mean } & \multirow{3}{*}{$\begin{array}{c}\text { Per cent } \\
\text { reducti } \\
\text { on over } \\
\text { UTC }\end{array}$} & \multirow{3}{*}{$\begin{array}{c}\text { No. of } \\
\text { thrips/inflo } \\
\text { rescence } \\
\text { after } 10 \\
\text { DAS of } \\
\text { second } \\
\text { spray }\end{array}$} \\
\hline & & \multirow[b]{2}{*}{ PTC } & \multicolumn{2}{|c|}{ First Spray } & \multicolumn{2}{|c|}{ Second Spray } & & & \\
\hline & & & 3 DAS & 10 DAS & 3 DAS & $\begin{array}{c}10 \\
\text { DAS }\end{array}$ & & & \\
\hline $\mathrm{T}_{1}$ & Azadirachtin 1500ppm @ 3.0 ml/1 & 10.22 & $\begin{array}{c}7.67 \\
(2.85) \mathrm{e}\end{array}$ & $\begin{array}{c}8.50 \\
(3.00) \mathrm{e}\end{array}$ & $\begin{array}{c}8.50 \\
(3.00) \mathrm{e}\end{array}$ & $\begin{array}{c}9.10 \\
(3.09) \mathrm{e}\end{array}$ & 8.44 & 33.80 & $\begin{array}{c}8.10 \\
(2.93) \mathrm{e}\end{array}$ \\
\hline $\mathrm{T}_{2}$ & NSKE 5\% @ 5.0 ml/1 & 10.78 & $\begin{array}{c}7.47 \\
(2.82) \mathrm{e}\end{array}$ & $\begin{array}{c}8.66 \\
(3.02) \mathrm{e}\end{array}$ & $\begin{array}{c}8.45 \\
(2.99) \mathrm{e}\end{array}$ & $\begin{array}{c}9.66 \\
(3.18) \mathrm{e}\end{array}$ & 8.56 & 32.86 & $\begin{array}{c}8.43 \\
(2.99) \mathrm{e}\end{array}$ \\
\hline $\mathrm{T}_{3}$ & Fipronil 5\%SC @ 1.0 ml/l & 9.62 & $\begin{array}{c}2.04 \\
(1.58) \mathrm{a}\end{array}$ & $\begin{array}{c}1.40 \\
(1.38) \mathrm{a}\end{array}$ & $\begin{array}{c}1.30 \\
(1.34) \mathrm{a}\end{array}$ & $\begin{array}{c}1.20 \\
(1.30) \mathrm{a}\end{array}$ & 1.48 & 88.39 & $\begin{array}{c}1.56 \\
(1.42) \mathrm{a}\end{array}$ \\
\hline $\mathrm{T}_{4}$ & Emamectin benzoate 5\%SG @ $0.2 \mathrm{~g} / 1$ & 10.40 & $\begin{array}{c}3.83 \\
(2.08) \mathrm{b}\end{array}$ & $\begin{array}{c}2.83 \\
(1.81) b\end{array}$ & $\begin{array}{c}3.33 \\
(1.95) \mathrm{b}\end{array}$ & $\begin{array}{c}3.03 \\
(1.87) \mathrm{b}\end{array}$ & 3.25 & 74.50 & $\begin{array}{c}3.00 \\
(1.87) b\end{array}$ \\
\hline $\mathrm{T}_{5}$ & Spinosad 45\%SC @ 0.25 ml/l & 10.00 & $\begin{array}{c}3.87 \\
(2.09) \mathrm{b}\end{array}$ & $\begin{array}{c}2.90 \\
(1.84) b\end{array}$ & $\begin{array}{c}3.66 \\
(2.04) b\end{array}$ & $\begin{array}{c}3.16 \\
(1.91) b\end{array}$ & 3.29 & 74.19 & $\begin{array}{c}3.46 \\
(1.99) \mathrm{b}\end{array}$ \\
\hline $\mathrm{T}_{6}$ & Imidacloprid 17.8\% SL @ 0.3 ml/1 & 9.40 & $\begin{array}{c}2.45 \\
(1.72) \mathrm{a}\end{array}$ & $\begin{array}{c}1.40 \\
(1.38) \mathrm{a}\end{array}$ & $\begin{array}{c}1.70 \\
(1.48) \mathrm{a}\end{array}$ & $\begin{array}{c}1.80 \\
(1.52) \mathrm{a}\end{array}$ & 1.83 & 85.64 & $\begin{array}{c}1.66 \\
(1.46) \mathrm{a}\end{array}$ \\
\hline $\mathrm{T}_{7}$ & Thiomethoxam 25\%WG @ 0.25 g/l & 9.62 & $\begin{array}{c}4.10 \\
(2.14) \mathrm{b}\end{array}$ & $\begin{array}{c}3.63 \\
(2.03) \mathrm{b}\end{array}$ & $\begin{array}{c}4.03 \\
(2.12) \mathrm{b}\end{array}$ & $\begin{array}{c}3.60 \\
(2.02) b\end{array}$ & 3.84 & 69.88 & $\begin{array}{c}3.70 \\
(2.05) b\end{array}$ \\
\hline $\mathrm{T}_{8}$ & Clothianidin 50\%WDG @ 0.2 g/l & 10.60 & $\begin{array}{c}5.13 \\
(2.37) \mathrm{c}\end{array}$ & $\begin{array}{c}5.31 \\
(2.41) \mathrm{c}\end{array}$ & $\begin{array}{c}5.21 \\
(2.39) \mathrm{c}\end{array}$ & $\begin{array}{c}5.41 \\
(2.43) \mathrm{c}\end{array}$ & 5.26 & 58.74 & $\begin{array}{c}4.63 \\
(2.26) c\end{array}$ \\
\hline $\mathrm{T}_{9}$ & Dimethoate 30\%EC @ 1 ml/l & 10.78 & $\begin{array}{c}6.30 \\
(2.60) \mathrm{d}\end{array}$ & $\begin{array}{c}6.63 \\
(2.67) d\end{array}$ & $\begin{array}{c}6.83 \\
(2.71) \mathrm{d}\end{array}$ & $\begin{array}{c}6.93 \\
(2.73) \mathrm{d}\end{array}$ & 6.67 & 47.68 & $\begin{array}{c}5.56 \\
(2.46) d\end{array}$ \\
\hline $\mathrm{T}_{10}$ & Lambda cyhalothrin 5\%EC @ 0.5 ml/1 & 10.50 & $\begin{array}{c}6.33 \\
(2.61) \mathrm{d}\end{array}$ & $\begin{array}{c}6.60 \\
(2.67) \mathrm{d}\end{array}$ & $\begin{array}{c}7.06 \\
(2.73) \mathrm{d}\end{array}$ & $\begin{array}{c}6.99 \\
(2.73) \mathrm{d}\end{array}$ & 6.75 & 47.05 & $\begin{array}{c}5.90 \\
(2.53) \mathrm{d}\end{array}$ \\
\hline $\mathrm{T}_{11}$ & Acephate 75\% SP @ 0.5 g/l & 9.52 & $\begin{array}{c}6.30 \\
(2.61) d\end{array}$ & $\begin{array}{c}6.70 \\
(2.68) d\end{array}$ & $\begin{array}{c}6.70 \\
(2.68) \mathrm{d}\end{array}$ & $\begin{array}{c}7.20 \\
(2.81) \mathrm{d}\end{array}$ & 6.72 & 47.29 & $\begin{array}{c}6.80 \\
(2.70) d\end{array}$ \\
\hline $\mathrm{T}_{12}$ & Untreated control & 9.56 & $\begin{array}{c}11.53 \\
(3.47) \mathrm{f}\end{array}$ & $\begin{array}{c}14.50 \\
(3.87) f\end{array}$ & $\begin{array}{c}13.20 \\
(3.70) \mathrm{f}\end{array}$ & $\begin{array}{l}11.80 \\
(3.51) \mathrm{f}\end{array}$ & 12.75 & - & $\begin{array}{l}11.43 \\
(3.45) f\end{array}$ \\
\hline & SE m \pm & NS & 0.07 & 0.09 & 0.08 & 0.08 & & & 0.08 \\
\hline & CD at $5 \%$ & NS & 0.20 & 0.25 & 0.24 & 0.25 & & & 0.24 \\
\hline & $\mathrm{CV}(\%)$ & NS & 5.21 & 6.20 & 5.93 & 6.18 & & & 5.93 \\
\hline
\end{tabular}

Figures in the parantheses are $\sqrt{\mathrm{x}+0.5}$ transformed values

Means followed by same letter do not differ significantly by DMRT $(\mathrm{P}=0.05)$

PTC - Pretreatment count per shoot

DAS - Day After Spraying

UTC - Untreated control

*Mean of thirty shoots 
Table.3 Efficacy of various insecticides on berry damage and grape fruit yield, 2013- 14

\begin{tabular}{|c|c|c|c|c|c|c|}
\hline \multirow[b]{2}{*}{$\begin{array}{l}\text { Tr. } \\
\text { No. }\end{array}$} & \multirow[b]{2}{*}{ Treatments } & \multirow[b]{2}{*}{$\begin{array}{c}\text { Per cent } \\
\text { berry } \\
\text { damage* }^{*}\end{array}$} & \multirow[b]{2}{*}{$\begin{array}{c}\text { Per cent } \\
\text { reduction } \\
\text { over } \\
\text { control }\end{array}$} & \multicolumn{3}{|c|}{ Fruit yield } \\
\hline & & & & $\begin{array}{c}\mathrm{Kg} / \\
\text { plant }\end{array}$ & t/ha & $\begin{array}{c}\text { Per cent } \\
\text { increase } \\
\text { over } \\
\text { UTC }\end{array}$ \\
\hline $\mathrm{T}_{1}$ & Azadirachtin 1500ppm @ 3.0 ml/1 & $56.43 \mathrm{e}$ & 18.15 & $6.83 \mathrm{e}$ & $23.69 \mathrm{e}$ & 17.18 \\
\hline $\mathrm{T}_{2}$ & NSKE 5\% @ $5.0 \mathrm{ml} / \mathrm{l}$ & $60.15 \mathrm{e}$ & 12.75 & $6.76 \mathrm{e}$ & $23.45 \mathrm{e}$ & 15.98 \\
\hline $\mathrm{T}_{3}$ & Fipronil 5\%SC @ 1.0 ml/1 & $21.89 \mathrm{a}$ & 68.25 & $12.13 \mathrm{a}$ & $42.08 \mathrm{a}$ & 108.11 \\
\hline $\mathrm{T}_{4}$ & Emamectin benzoate 5\%SG @ $0.2 \mathrm{~g} / 1$ & $30.81 \mathrm{~b}$ & 55.31 & $11.06 \mathrm{~b}$ & $38.37 \mathrm{~b}$ & 89.75 \\
\hline $\mathrm{T}_{5}$ & Spinosad 45\%SC @ 0.25 ml/1 & $31.34 \mathrm{~b}$ & 54.54 & $10.83 \mathrm{~b}$ & $37.57 \mathrm{~b}$ & 85.81 \\
\hline $\mathrm{T}_{6}$ & Imidacloprid 17.8\%SL @ 0.3 ml/1 & $24.60 \mathrm{a}$ & 64.32 & $12.00 \mathrm{a}$ & $41.63 \mathrm{a}$ & 105.88 \\
\hline $\mathrm{T}_{7}$ & Thiomethoxam 25\%WG @ $0.25 \mathrm{~g} / \mathrm{l}$ & $33.70 \mathrm{~b}$ & 51.12 & $10.73 \mathrm{~b}$ & $37.22 \mathrm{~b}$ & 84.09 \\
\hline $\mathrm{T}_{8}$ & Clothianidin 50\%WDG @ 0.2g/1 & $38.34 \mathrm{c}$ & 44.39 & $9.26 \mathrm{c}$ & $32.12 \mathrm{c}$ & 58.87 \\
\hline $\mathrm{T}_{9}$ & Dimethoate 30\%EC @ 1 ml/1 & $48.94 \mathrm{~d}$ & 29.01 & $8.33 \mathrm{~d}$ & $28.90 \mathrm{~d}$ & 42.92 \\
\hline $\mathrm{T}_{10}$ & Lambda cyhalothrin 5\%EC @ $0.5 \mathrm{ml} / 1$ & $49.49 \mathrm{~d}$ & 28.21 & $8.30 \mathrm{~d}$ & $28.79 \mathrm{~d}$ & 42.40 \\
\hline $\mathrm{T}_{11}$ & Acephate 75\% SP @ 0.5 g/l & $47.92 \mathrm{~d}$ & 30.49 & $7.70 \mathrm{~d}$ & $26.71 \mathrm{~d}$ & 32.11 \\
\hline $\mathrm{T}_{12}$ & Untreated control & $68.94 \mathrm{f}$ & - & $5.83 \mathrm{f}$ & $20.22 \mathrm{f}$ & - \\
\hline & $\mathrm{SE} \mathrm{m} \pm$ & 1.48 & & 0.28 & 0.291 & \\
\hline & $\mathrm{CD}$ at $5 \%$ & 4.42 & & 0.86 & 0.87 & \\
\hline & $\mathrm{CV}(\%)$ & 6.12 & & 5.58 & 5.60 & \\
\hline
\end{tabular}

PTC - Pretreatment count per shoot

DAS - Day After Spraying

UTC - Untreated control

*Mean of thirty bunches

Table.4 Economics of management of thrips on grapes

\begin{tabular}{|c|c|c|c|c|c|c|c|c|c|}
\hline $\begin{array}{l}\text { Tr. } \\
\text { No. }\end{array}$ & Treatements & Dosage $\mathbf{~ m l} / \mathrm{g}$ & $\begin{array}{c}\text { Yield / ha } \\
\text { (tonnes) }\end{array}$ & $\begin{array}{c}\text { Gross } \\
\text { Returns } \\
\text { (Rs./ha) }\end{array}$ & $\begin{array}{l}\text { Incremental } \\
\text { yield over } \\
\text { control (t/ha) }\end{array}$ & $\begin{array}{c}\text { Incremental } \\
\text { Benefit over } \\
\text { control } \\
\text { (Rs/ha) }\end{array}$ & $\begin{array}{c}* \text { Cost of } \\
\text { plant } \\
\text { protectio } \\
\text { n }\end{array}$ & $\begin{array}{l}\text { Additional } \\
\text { Net profit }\end{array}$ & $\begin{array}{c}\text { IBC } \\
\mathbf{R}\end{array}$ \\
\hline $\mathrm{T}_{1}$ & Azadiracthin 1500ppm & $3.0 \mathrm{ml} / \mathrm{l}$ & 23.69 & 568560 & 3.47 & 83280 & 12775 & 70505 & 5.51 \\
\hline $\mathrm{T}_{2}$ & NSKE 5\% & $5.0 \mathrm{ml} / \mathrm{l}$ & 23.45 & 562800 & 3.23 & 77520 & 3090 & 74430 & 24.08 \\
\hline $\mathrm{T}_{3}$ & Fipronil 5SC & $0.1 \mathrm{ml} / \mathrm{l}$ & 42.08 & 1009920 & 21.86 & 524640 & 29261 & 521713 & 16.92 \\
\hline $\mathrm{T}_{4}$ & Emamectin benzoate 5SG & $0.2 \mathrm{~g} / \mathrm{l}$ & 38.37 & 920880 & 18.15 & 435600 & 12030 & 423570 & 35.20 \\
\hline $\mathrm{T}_{5}$ & Spinosad 45SC & $0.25 \mathrm{ml} / \mathrm{l}$ & 37.57 & 901680 & 17.35 & 416400 & 27673 & 388726 & 14.04 \\
\hline $\mathrm{T}_{6}$ & Imidacloprid 200SL & $0.3 \mathrm{~g} / \mathrm{l}$ & 41.63 & 999120 & 21.41 & 513840 & 6857 & 506982 & 73.93 \\
\hline $\mathrm{T}_{7}$ & Thiomethoxam 25WG & $0.25 \mathrm{~g} / 1$ & 37.22 & 893280 & 17 & 408000 & 54370 & 353630 & 6.50 \\
\hline $\mathrm{T}_{8}$ & Clothianidin 50\%WDG & $0.2 \mathrm{~g} / \mathrm{l}$ & 32.12 & 770880 & 11.9 & 285600 & 57472 & 228128 & 3.96 \\
\hline $\mathrm{T}_{9}$ & Dimethoate 30EC & $1 \mathrm{ml} / 1$ & 28.9 & 693600 & 8.68 & 208320 & 5325 & 202995 & 38.12 \\
\hline $\mathrm{T}_{10}$ & Lambda Cyhalothrin 5EC & $0.5 \mathrm{ml} / 1$ & 28.79 & 690960 & 8.57 & 205680 & 3984 & 201696 & 50.62 \\
\hline $\mathrm{T}_{11}$ & Acephate $75 \mathrm{SP}$ & $0.5 \mathrm{~g} / \mathrm{l}$ & 26.71 & 641040 & 6.49 & 155760 & 3984 & 151776 & 38.09 \\
\hline $\mathrm{T}_{12}$ & Untreated control & - & 20.22 & 485280 & - & - & - & - & \\
\hline \multicolumn{2}{|c|}{ SEm \pm} & & 0.204 & & & & & & \\
\hline \multicolumn{2}{|c|}{$\mathrm{CV}(5 \%)$} & & 5.376 & & & & & & \\
\hline \multicolumn{2}{|c|}{$\mathrm{CD}$ at $5 \%$} & & 0.968 & & & & & & \\
\hline
\end{tabular}

IBCR=Incremental Cost Benefit Cost Ratio, *Cost of treatment + Cost of application *Market price of Grape was Rs.24/kg. Azadiractin, NSKE 10Kg: Rs.400, Fipronil 5\% SC 250ml: Rs.280, Emamectin benzoate 5 SG 100g: Rs.700, Spinosad 45 SC 75ml: Rs.1050, Imidacloprid 200 SL 50ml: Rs.140, Thiomethoxam 25 WG 12gm: Rs.340, Clothianidin 50\% WDG 6g: Rs.90, Dimethoate 30 EC 100ml: Rs.50, Lambda cyhalothrin 5 EC 250ml: Rs.160, Acephate 75 SP 250g: Rs.160. 


\section{Effect on the yield and economics}

Yield losses due to $S$. dorsalis were significantly low in all insecticides treatment over farmers practice. The data pertaining to fruit yield (Table 3) revealed that, significantly highest yield was obtained in $\mathrm{T}_{3}$ (42.08 t/ha) and $\mathrm{T}_{6}(41.63 \mathrm{t} / \mathrm{ha})$. These results are in agreement with Sunitha et al., (2008), who reported significantly highest fruit yield of grapes in neonicotinoid treatments viz., imidacloprid, acetamaprid, thiaomethoxam. The next best treatments were $\mathrm{T}_{4}(38.37 \mathrm{t} / \mathrm{ha})$, $\mathrm{T}_{5}$ (37.57 t/ha) and $\mathrm{T}_{7}$ (37.22 $\left.\mathrm{t} / \mathrm{ha}\right)$, followed by $\mathrm{T}_{8}(32.12 \mathrm{t} / \mathrm{ha})$. Among botanical products $\mathrm{T}_{1}(23.69 \mathrm{t} / \mathrm{ha})$ and $\mathrm{T}_{2}(23.45 \mathrm{t} / \mathrm{ha})$ were significantly superior over untreated control (20.22 t/ha). Keshav and Veer singh (2013) also reported ineffectiveness of NSKE and azadirachtin in decreasing the fruit yield in garlic ecosystem which agrees with the present findings.

Table 4 shows the cost of economics was worked out for various treatments used in the management of thrips and are presented. The higher gross returns were obtained from the treatment fipronil $5 \% \mathrm{SC}$ at $1.00 \mathrm{ml} / \mathrm{l}$ of water and imidacloprid $17.8 \% 0.3 \mathrm{ml} / \mathrm{l}$ of water (Rs. $10,09,9,20$ and $9,99,120$ respectively). The net returns were highest in the treatments fipronil $5 \% \mathrm{SC}$ at $1.00 \mathrm{ml} / \mathrm{l}$ of water and imidacloprid $17.8 \% 0.3 \mathrm{ml} / \mathrm{l}$ of water (Rs. $5,24,640$ and 5,13,840/ha respectively).

Highest IBCR of 73.93 was obtained from the treatment imidacloprid $17.8 \% \quad 0.3 \mathrm{ml} / \mathrm{l}$ of water (73.93) followed by thiomethoxam 25 WG $0.25 \mathrm{~g} / \mathrm{l}$ of water (58.32), lambda cyhalothrin 5\% EC @ $0.5 \mathrm{ml} / 1$ of water (50.62), fipronil $5 \% \mathrm{SC}$ at $1.00 \mathrm{ml} / \mathrm{l}$ of water (42.81). Even though higher benefits were obtained from the treatments emamectin benzoate 5\% SG @ $0.2 \mathrm{~g} / \mathrm{l}$ of water and spinosad 45 SC @ $0.25 \mathrm{ml} / 1$ of water, ICBR was low due to higher market price of insecticides.

\section{References}

Anonymous, 2012, Package of Practice for fruit crops, UHS, Bagalkot.

Anonymous, 2013, Grapes. Indian Horticulture Database 2013, Kumar, B., Mistry, N.C., Singh, B. and Gandhi, C.P. (Eds), National Horticulture Board, Gurgaon, India, pp. 68-75.

Atwal, A. S., and Dhaliwal, G. S., 2005, Agricultural Pests of South Asia and their Management. Kalyani Publishers, New Delhi, pp. 307-308.

Bhargava, K. K., and Bhatnagar, A., 2005, Field evaluation of conventional and eco-friendly insecticides against onion thrips, $\quad T$. tabaci (Lindeman) (Thysanoptera: Thripidae), Udyaika, 11 :73-78.

Bose, T. K., Mitra, S. K., A. K., Farooqi, A. A. and Sadhu, M. K., 1999, Grapes. Trop. Horti., Naya Prokash, Calcutta, India, pp. 259-268.

Bournier, A., 1977, Les Thrips : Biologie, Importance Agronomique. INRA, Paris, pp. 25-28.

Gomez, K. A., and Gomez, A. A., 1984, Statistical Procedures for Agricultural research. New Delhi, Wiley International Sciences Publication. 368 pp.

Jyothi, P., Ashoka, J., Bheemanna, M., Sreenivas, A. G., Naganagoud, A. and Narayan Rao, K., 2013, Management of thrips, Pseudodendrothrips mori (Niwa) using insecticides and botanical (Nimbicidine) J. Ent. Res., 37 (3): 207209.

Kanara, H. G., and Acharya, M. F., 2014, Evaluation of new insecticides against western flower thrips, Frankliniella occidentalis Pergande on Rose in vivo condition. Pestology, 38 (1)

Keshav, M., and Veer singh, 2013, Bioefficacy of New Insecticides against Thrips tabaci Lindeman on Garlic, Indian J. Entomology, 75 (3): 239-267. 
Sunitha, N. D., and Jagginavar, S. B., 2008, Management for Thrip complex in Grape Ecosystem. Ann. Pl. Prot. Sci., 16 (1): 83-86.

Suresh., Rajavel, D. S., Baskaran, R. K. and Rani, B. U., 2006, In vivo evaluation of various botanicals against onion thrips, Thrips tabaci (Lindeman) and cutworm, Agrotis ipsilon (Hufn.), Hexapoda., 13 : 47-52.

\section{How to cite this article:}

Nagaraj R. Patil, Nadaf, A.M., B.S. Sagar and Sahithya, B.R. 2017. Field Efficacy of Newer Insecticides and Neem Products against Scirtothrips dorsalis on Grapes, Vitis vinifera L. (cv. Thompson Seedless). Int.J.Curr.Microbiol.App.Sci. 6(9): 3287-3294. doi: https://doi.org/10.20546/ijcmas.2017.609.405 